\title{
Arterial oxygenation does not predict the outcome of patients with acute respiratory failure
}

\author{
P. Jiménez, A. Torres, J. Roca, A. Cobos, R. Rodriguez-Roisin
}

\begin{abstract}
Arterial oxygenation does not predict the outcome of patients with acute respiratory failure needing mechanical ventilation. P. Jiménez, A. Torres, J. Roca, A. Cobos, R. Rodriguez-Roisin. CERS Journals Ltd 1994.

ABSTRACT: One hundred and eighteen patients consecutively submitted to mechanical ventilation during a one year period, and admitted to the Respiratory Intensive Care Unit of the Hospital Clinic of Barcelona, were prospectively studied in order to define the importance of hypoxaemia as a predictor of mortality.

Using a discriminant multivariate analysis, the following variables were selected as the best predictors of outcome: 1) the number of associated complications (NAC) on admission; 2) the simplified acute physiologic score (SAPS); 3) oxygenation index $\left(\mathrm{PAO}_{2}-\mathrm{AaPO}_{2}\right) / \mathrm{PAO}_{2}+\mathbf{0 . 0 1 4}$ positive end-expiratory pressure (PEEP); and 4) the age of the patients.

Using these predictors, $84 \%$ of the patients were accurately classified as survivors or as nonsurvivors. The partial contribution of each predictor to the model was also assessed using a logistic regression, by eliminating each single predictor and each possible pair of predictors. Using this means of analysis, the NAC and SAPS were the only predictors of mortality. The inclusion of short-term mechanicallyventilated patients did not bias the accuracy of prediction.

Eur Respir J., 1994, 7, 730-735.
\end{abstract}

Servei de Pneumologia i Allèrgia Respiratòria, Hospital Clinic de Barcelona, Barcelona, Spain.

\section{Correspondence: A. Torres}

Servei de Pneumologia i Allèrgia Respiratòria Hospital Clinic

Villarroel 170

08036 Barcelona

Spain

Keywords: Discriminant analysis prognosis

severe pulmonary insufficiency

Received: June 101993

Accepted after revision November 211993
The decision to initiate mechanical ventilation is usually guided by several clinical parameters reflecting the disturbances of gas exchange and/or pulmonary mechanics [1]. The importance of these physiological alterations on the outcome of patients in acute respiratory failure requiring mechanical ventilation has not, as yet, been fully defined. Predictors of mortality have been studied in some populations. For instance, in patients with adult respiratory distress syndrome (ARDS), mortality depends, in particular, on the presence or development of a sepsis syndrome and/or pulmonary infection [2-4]. Alternatively, in patients with chronic obstructive pulmonary disease (COPD), the outcome seems to be related to the degree of respiratory impairment and to the presence of right heart failure [5-10].

The present study was designed to investigate the predictors of outcome in a cohort of patients admitted to a Respiratory Intensive Care Unit (RICU) needing mechanical ventilation. The following questions were specifically addressed: 1) What is the role of hypoxaemia and derived parameters in predicting mortality? 2) Are the clinical and laboratory data collected during the first hours of admission useful to establish a reliable prognosis? and 3) Can the inclusion of short-term mechanically-ventilated patients bias the prediction of outcome?

\begin{abstract}
Methods
The study was conducted at the Respiratory Intensive Care Unit of the Hospital Clinic de Barcelona, a 1,000 bed teaching hospital. All consecutively admitted patients who needed mechanical ventilation at our RICU over a one year period were included in the study. All patients were connected to a Servo 900-C (Siemens Elema, Sweden) ventilator.

On admission to the RICU and after mechanical ventilation was instituted, the following variables were recorded: age, sex, arterial blood gases (ABG), ventilatory settings (inspiratory oxygen fraction $\left(\mathrm{FIO}_{2}\right)$, respiratory frequency, tidal volume, peak and plateau pressures, and positive end-expiratory pressure (PEEP), the simplified acute physiologic score (SAPS) and plasma sodium and creatinine levels. Data were stored in a computer data base.

All ABG measurements were obtained under identical uniform conditions: controlled mechanical ventilation, PEEP $5 \mathrm{cmH}_{2} \mathrm{O}$ in ARDS patients, $\mathrm{FIO}_{2} 1.0$, tidal volume $10 \mathrm{ml} \cdot \mathrm{kg}^{-1}$, respiratory rate $10-14$ breaths $\cdot \mathrm{min}^{-1}$, and an inspiratory/expiratory $(\mathrm{I} / \mathrm{E})$ ratio $1 / 2$. These ventilatory settings were different only for patients with status asthmaticus, in whom we used a controlled hypoventilation
\end{abstract}


with an $\mathrm{I} / \mathrm{E}$ ratio of $1 / 3$. From the $\mathrm{ABG}$ data, the following parameters were calculated: arterial oxygen tension $\left(\mathrm{PaO}_{2}\right)$ /alveolar oxygen tension $\left(\mathrm{PAO}_{2}\right), \mathrm{PaO}_{2} / \mathrm{FIO}_{2}$, alveolar arterial oxygen tension gradient $\left(\mathrm{AaPo}_{2}\right)$ (the $\mathrm{AaPO}_{2}$ difference was calculated using the standard alveolar air equation assuming an $\mathrm{R}$ equal to 0.8 ), $\mathrm{AaPO}_{2} / \mathrm{FIO}_{2}$, and the oxygenation index (OI) modified from JARDIN et al. [10] $\left(\left(\mathrm{PAO}_{2}-\mathrm{AaPO}_{2} / \mathrm{PaO}_{2}\right)+(0.014 \times \mathrm{PEEP})\right)$ [1]. From ventilatory settings, minute volume, dynamic compliance (tidal volume (VT)/peak pressure(Ppeak)-PEEP) and static compliance (VT/plateau pressure(Pplat)-PEEP) were measured.

The presence of associated complications on admission was recorded according to the following definitions: 1) "respiratory infection" was defined as the presence of fever, purulent sputum and leucocytosis, associated with new and persistent radiographic infiltrates [11]; 2) "sepsis syndrome" was defined as positive blood cultures or fever $>39^{\circ} \mathrm{C}$, together with positive cultures from suspected sources (urine, abscess, draining wound, ascites or pleural fluid) [12]; 3) "shock" was defined as systolic hypotension lower than $90 \mathrm{mmHg}$ with decreased urinary output, or hypotension requiring vasoactive amines infusion to obtain blood pressure of $\geq 90 \mathrm{mmHg}$ [13]; 4) "renal failure" was defined as an acute rise in serum creatinine $>265.2 \mu \mathrm{mol} \cdot l^{-1}$ in patients with previously normal renal function [12]; 5) "cardiac impairment" included arrhythmia requiring emergency therapeutic intervention, or tachycardia along with clinical and radiographic evidence of pulmonary congestion which resolved with diuretic therapy, or a decreased cardiac index with increased wedge pressure [12]; 6) "disseminated intravascular coagulation" was defined as the presence of thrombocytopenia $(<80$ platelets $\times 10^{3} \cdot \mathrm{mm}^{-3}$ ), increased prothrombin time, increased fibrinogen degradation products, or decreased fibrinogen levels [12]; 7) "gastrointestinal complications" included macroscopic digestive bleeding, or intestinal perforation or acute intestinal ischaemia demonstrated by laparotomy, or pancreatitis with a twofold increase in amylase or lipase enzymes, or hepatic dysfunction defined as a twofold increase of glutamic pyruvic transaminase or alkaline phosphatase or development of jaundice [12]; 8) "coma" was defined as a Glasgow coma score $<10$ [14]; 9) "metabolic complications" included hyponatraemia $<120 \mathrm{mmol} \cdot l^{-1}$, or serum potassium $<3.0 \mathrm{mmol} \cdot l^{-1}$ or $>6.0 \mathrm{mmol} \cdot l^{-1}$, or hypercalcaemia, or alkalosis with $\mathrm{pH}>7.60$, or metabolic acidosis $(\mathrm{pH}<7.20)$. The resultant score was the addition of the number of associated complications (NAC). The simplified acute physiological score (SAPS) described by LE GALL et al. [15] was calculated without including the Glasgow coma score due to intubation and sedation of the patients.

The variables referred to above were recorded on admission, and at 24 and $72 \mathrm{~h}$ for ventilated patients. Survival was defined as still alive at discharge from the RICU.

\section{Statistical analysis}

Data are expressed as mean $\pm \mathrm{SD}$.

\section{Univariate analysis}

The variables described above were compared between survivor and nonsurvivor populations by means of analysis of variance. This analysis was performed for the whole population, for patients needing mechanical ventilation more than $24 \mathrm{~h}$, and for those patients requiring mechanical ventilation more than $72 \mathrm{~h}$.

\section{Multivariate analysis}

A discriminant analysis was performed in order to classify the cases according to the independent variables recorded. Discriminant analysis allows the classification of the population under study into groups defined by a binary dependent variable (in this case survival/death). The independent variables are included stepwise into a linear equation until the best combination of variables for classification is obtained [16].

The statistical analysis was performed as follows. Firstly, discriminant analysis included the overall cohort (118 patients); the independent variables analysed were: age, number of associated complications on admission, $\mathrm{PaO}_{2} / \mathrm{PAO}_{2}, \mathrm{PaO}_{2} / \mathrm{FIO}_{2}, \mathrm{AaPo}_{2}$ gradient, $\mathrm{AaPO}_{2}$ gradient/ $\mathrm{FIO}_{2}$, OI, minute ventilation, dynamic and static compliance and SAPS. Secondly, the correlation matrix was examined, and in the case of highly correlated variables (i.e $r>0.6$ ), only those with the higher significance in the analysis of variance were selected for further analysis. A subsequent discriminant analysis, excluding the highly correlated variables, was then performed. Finally, to assess the influence of gas exchange variables on the outcome, a third discriminant analysis was performed excluding the ABG data. Receiver operating characteristic (ROC) curves were obtained from the last two discriminant functions, to evaluate the loss in discriminant ability of the function after removing ABG data. Since ROC curves were not obtained from a different "validation" sample but from the same sample used to derive the corresponding discriminant functions, it is likely that both curves overestimate the discriminant ability of these functions. The partial contribution of each predictor (including the ABG data) to the model was assessed using logistic regression, fitting more parsimonious models by elimination: 1) each single predictor at a time; and 2) each possible pair of predictors. The drop in the fit was then evaluated as the difference in degrees of freedom, using the Chi-squared distribution.

In order to assess the influence and possible bias of the inclusion of short-term mechanical ventilation population in predicting outcome, similar analyses were performed in the population requiring mechanical ventilation more than $24 \mathrm{~h}$ and more than $72 \mathrm{~h}$.

\section{Results}

One hundred and twenty three patients were submitted to mechanical ventilation in our RICU during the study period. Five of these were excluded because of incomplete records. Clinical conditions leading to mechanical 
ventilation in the remaining 118 patients are shown in table 1 . Fourteen patients were weaned from mechanical ventilation during the first $24 \mathrm{~h}$, and 28 of the 104 remaining patients were weaned during the next $48 \mathrm{~h}$. Thus, 76 patients continued to be on mechanical ventilation $72 \mathrm{~h}$ following their admission.

Thirty three patients from the whole group $(n=118)$ died. Thirty out of these 33 required ventilation for more than $24 \mathrm{~h}$, and twenty five were ventilated for more than $72 \mathrm{~h}$.

The variables studied that showed significant differences regarding mortality are shown in table 2 . The survival group had less severe acute respiratory failure, lower SAPS score, fewer NAC, and an older age than the nonsurvival group, on admission and thereafter.

\section{Multivariate analysis}

The variables with significant differences at the univariate analysis were included in a first discriminant analysis studying all the population submitted to mechanical

Table 1. - Conditions leading to mechanical ventilation

\begin{tabular}{lcc}
\hline & $\mathrm{n}$ & $\%$ \\
\hline Postsurgical & 30 & 25 \\
Pneumonia & 22 & 19 \\
Acute exacerbation of CRF & 19 & 16 \\
Multiple trauma & 12 & 10 \\
Acute pulmonary oedema & 9 & 8 \\
Cardiorespiratory arrest & 8 & 7 \\
Status asthmaticus & 7 & 6 \\
Alveolar hypoventilation syndrome & 6 & 5 \\
Miscellaneous & 5 & 4 \\
\hline Total & $118^{*}$ & \\
\hline
\end{tabular}

*: 10 of these 118 patients met criteria of adult respiratory distress syndrome (ARDS). CRF: chronic respiratory failure. ventilation. The NAC on admission, the SAPS, the OI, and the age of patients were selected as the best combination of predictors. When the OI was excluded from the analysis, the classification did not change. The overall accuracy of the classification was $84 \%$, including a positive predictive value for survival of $84 \%$ in both analyses (table 3 ). Figure $1 \mathrm{a}$ and $1 \mathrm{~b}$ shows ROC curves associated to the discriminant functions including: a) NAC, SAPS, age and OI; and b) NAC, SAPS and age. The two curves are very similar, indicating that dropping OI from the model did not result in a relevant worsening in the prediction of the outcome.

One hundred and four patients needed mechanical ventilation for more than $24 \mathrm{~h}$ after RICU admission, of whom 30 patients $(29 \%)$ died. The discriminant analysis performed in this subsample showed similar results to those obtained from the initial 118 patients studied. Again, when excluding the OI from the analysis, the classification did not change. The overall accuracy was $82 \%$, with a positive predictive value for survival of $83 \%$.

Seventy two hours after admission to the RICU, 43 patients had been weaned from mechanical ventilation, so that 76 patients remained mechanically-ventilated. Twenty five individuals (33\%) of the latter group died. Again we obtained similar results to those mentioned above. Sixty two out of 76 cases $(82 \%)$ were correctly classified using the variables selected (NAC+SAPS+age+ OI). When the OI was excluded from the analysis, the overall accuracy was $79 \%$ (60 out of 75 cases).

Table 4 shows the results of the logistic regression analysis for the overall population $(n=118)$. The first model with the four predictors (NAC+SAPS+age+OI) shows reasonably good fit in all three groups (all patients, $\mathrm{MV}>24 \mathrm{~h}$ and MV $>72 \mathrm{~h}$ ). Excluding either age or OI from the initial model did not result in a significant worsening of fit ( $p$-value associated to the difference of fit $(\mathrm{p}(\mathrm{d})>0.05)$. However, those models excluding either

Table 2. - Results of univariate analysis in patients submitted to mechanical ventilation (MV): comparison of survivors and nonsurvivors

\begin{tabular}{|c|c|c|c|c|c|c|c|}
\hline \multirow[t]{2}{*}{ Variables } & \multirow[t]{2}{*}{ Survival } & \multirow{2}{*}{$\begin{array}{l}\text { All patients } \\
\mathrm{n}=123\end{array}$} & \multirow[b]{2}{*}{$\mathrm{p}^{*}$} & \multicolumn{2}{|c|}{ MV > 24 h patients } & \multicolumn{2}{|c|}{$\mathrm{MV}>72 \mathrm{~h}$ patients } \\
\hline & & & & $\mathrm{n}=30$ & $\mathrm{p}^{*}$ & $\mathrm{n}=25$ & $\mathrm{p}^{*}$ \\
\hline \multirow[t]{2}{*}{ Age yrs } & Yes & $59 \pm 15$ & & $58 \pm 15$ & & $59 \pm 14$ & \\
\hline & No & $49 \pm 20$ & 0.0053 & $48 \pm 20$ & 0.0024 & $46 \pm 22$ & 0.0027 \\
\hline \multirow[t]{2}{*}{$\mathrm{PaO}_{2} / \mathrm{FIO}_{2} \quad \mathrm{kPa}$} & Yes & $40 \pm 19$ & & $38 \pm 14$ & & $38 \pm 13$ & \\
\hline & No & $31 \pm 18$ & 0.0264 & $32 \pm 13$ & 0.0011 & $26 \pm 12$ & 0.0001 \\
\hline \multirow[t]{2}{*}{$\mathrm{PaO}_{2} / \mathrm{PAO}_{2} \%$} & Yes & $50 \pm 20$ & & $50 \pm 20$ & & $50 \pm 20$ & \\
\hline & No & $38 \pm 20$ & 0.0066 & $40 \pm 20$ & 0.0004 & $32 \pm 17$ & 0.0001 \\
\hline \multirow[t]{2}{*}{$\mathrm{AaPO}_{2} / \mathrm{FIO}_{2} \quad \mathrm{kPa}$} & Yes & $40 \pm 18$ & & $41 \pm 16$ & & $38 \pm 15$ & \\
\hline & No & $50 \pm 18$ & 0.0084 & $54 \pm 15$ & 0.0001 & $56 \pm 16$ & 0.0001 \\
\hline \multirow[t]{2}{*}{$\mathrm{OI} * *$} & Yes & $51 \pm 21$ & & $53 \pm 21$ & & $52 \pm 19$ & \\
\hline & No & $62 \pm 20$ & 0.0005 & $68 \pm 20$ & 0.0008 & $72 \pm 22$ & 0.0002 \\
\hline \multirow[t]{2}{*}{ SAPS } & Yes & $14 \pm 3$ & & $10 \pm 3$ & & $10 \pm 3$ & \\
\hline & No & $18 \pm 6$ & 0.0001 & $13 \pm 4$ & 0.0001 & $13 \pm 4$ & 0.0001 \\
\hline \multirow{2}{*}{ NAC } & Yes & $0.7 \pm 0.6$ & & $0.7 \pm 0.6$ & & $0.7 \pm 0.6$ & \\
\hline & No & $1.9 \pm 1.3$ & 0.0001 & $1.8 \pm 1.2$ & 0.0001 & $1.7 \pm 1.3$ & 0.0001 \\
\hline
\end{tabular}

Data are presented as mean \pm SD. *: level of significance at analysis of variance; **: oxygenation index $(\mathrm{OI})=\left(\left(\mathrm{PAO}_{2}-\mathrm{AaPO}_{2}\right.\right.$ gradient $\left./ \mathrm{PAO}_{2}\right)$ $+(0.014 \times \mathrm{PEEP})) . \quad \mathrm{PaO}_{2}$ : arterial oxygen tension; $\mathrm{FIO}_{2}$ : inspiratory oxygen fraction; $\mathrm{PAO}_{2}$ : alveolar oxygen tension; AaPo$:$ alveolar arterial oxygen tension gradient; SAPS: simplified acute physiological score; NAC: number of associated complications. 
Table 3. - Classification of the outcome in 118 patients submitted to mechanical ventilation according to discriminant function, with and without inclusion of oxygenation index

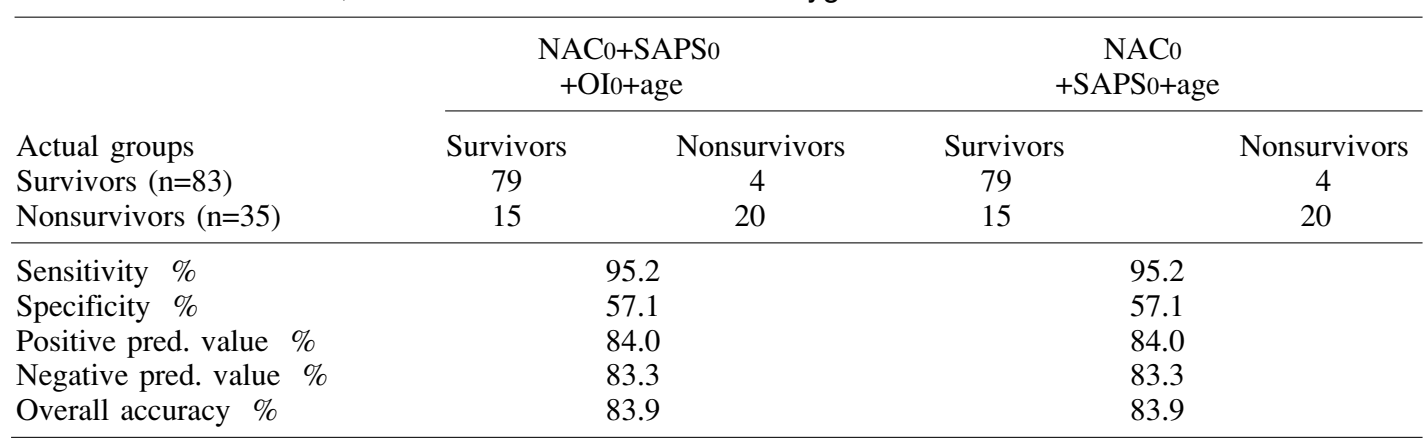

NAC0: number of associated complications on admission; SAPS0: simplified acute physiological score (version of APACHE score) on admission; OI0: oxygenation index on admission; pred.: predictive.

a)

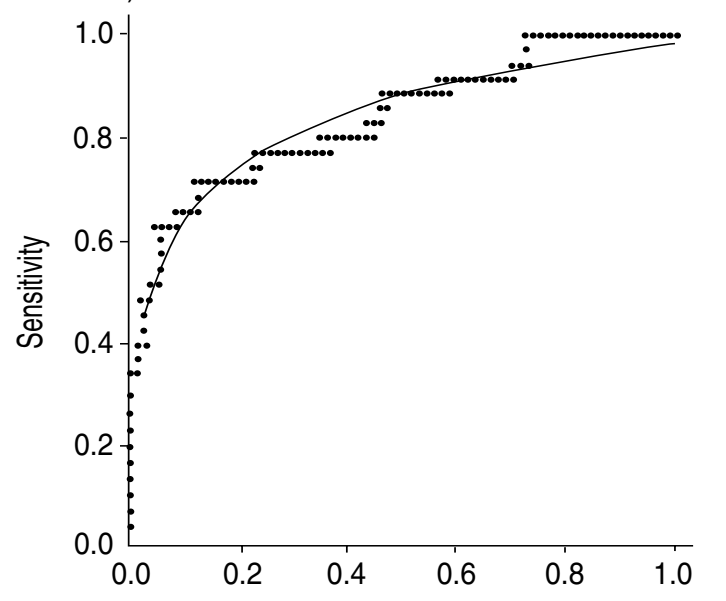

b)

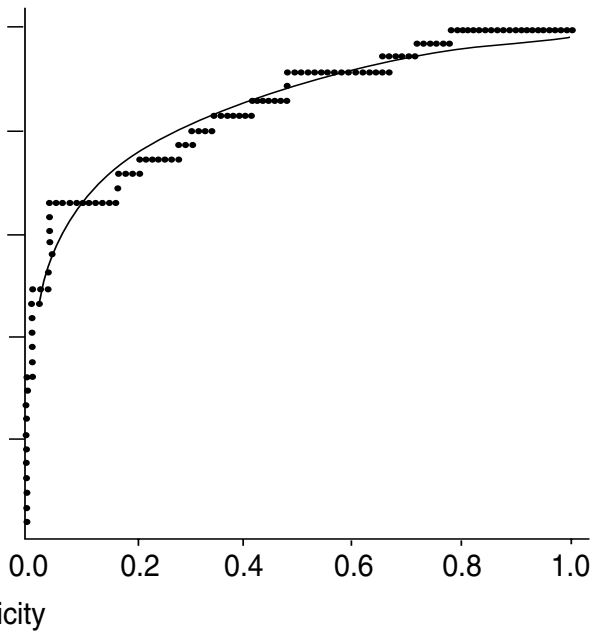

Fig. 1. - ROC curves associated to discriminant function including: a) NAC+SAPS+age+OI; and b) NAC+SAPS+age, as predictor variables. NAC: number of associated complications; SAPS: simplified acute physiological score; OI: oxygenation index; ROC: receiver operating characteristic.

Table 4. - Goodness-of-fit test for logistic regression models

\begin{tabular}{lrccc}
\hline & \multicolumn{4}{c}{ All patients (n=118) } \\
\cline { 2 - 5 } Variables in the model & \multicolumn{1}{c}{$\mathrm{L}$} & $\mathrm{df}$ & $\mathrm{p}$ & $\mathrm{p}(\mathrm{d})$ \\
\hline SAPS, NAC, OI, age & 95.24 & 113 & 0.8858 & \\
SAPS, NAC, OI & 97.19 & 114 & 0.8705 & 0.1629 \\
SAPS, NAC, age & 95.50 & 114 & 0.8950 & 0.6101 \\
SAPS, OI, age & 114.23 & 114 & 0.4762 & 0.0000 \\
NAC, OI, age & 103.13 & 114 & 0.7579 & 0.0050 \\
SAPS, NAC & 97.67 & 115 & 0.8773 & 0.2976 \\
SAPS, OI & 117.20 & 115 & 0.4253 & 0.0000 \\
SAPS, age & 117.57 & 115 & 0.4161 & 0.0000 \\
NAC, OI & 106.31 & 115 & 0.7067 & 0.0039 \\
NAC, age & 103.84 & 115 & 0.7633 & 0.0136 \\
OI, age & 130.93 & 115 & 0.1471 & 0.0000 \\
\hline
\end{tabular}

L: -2 log-likelihood ratio (statistic to assess the overall fit of the model); df: degrees of freedom; $p$ : p-value of the goodness of fit test (the higher the $p$, the better the goodness of fit); $p(d)$ : $\mathrm{p}$-value associated to the difference of fit between the model with four predictor variables and the current model (values below 0.05 indicate a significant worsening in fit with respect to the model including four predictors. For further abbreviations see legend to table 2 .
SAPS or NAC, or both, show a significantly worse fit $(p(d)<0.05)$. This pattern of results was stable among all three groups considered.

\section{Discussion}

Our results show that the severity of acute illness is the main condition influencing the outcome in patients requiring mechanical ventilation. This conclusion was assessed studying the number of associated complications on admission to our RICU and also by sequential measurements of the simplified acute physiologic score. By contrast, variables reflecting the degree of severity of respiratory failure, such as the $\mathrm{PaO}_{2} / \mathrm{PAO}_{2}, \mathrm{PaO}_{2} / \mathrm{FIO}_{2}$ and $\mathrm{AaPO}_{2} / \mathrm{FIO}_{2}$ ratios, the $\mathrm{AaPO}_{2}$ and the $\mathrm{OI}$ had a poor predictive value to determine the outcome. A logistic regression analysis excluding each of the initial predictors also demonstrated that both SAPS and NAC were the only predictors of mortality. Identical results were obtained when patients mechanically-ventilated at 24 and $72 \mathrm{~h}$ were considered.

The main results (SAPS and NAC as negative predictors and age and hypoxaemia as nonpredictors) of the present study partially agree with other studies regarding 
mechanically-ventilated patients. For instance, KNAUS [17], investigating prognosis factors of 571 patients, found that prognosis varies with the acute indication for mechanical ventilation, the severity of the disease, and the patient's physiological reserve determined by age and chronic health status.

Almost all the variables analysed in the present study showed significant differences in the univariate analysis between survivors and nonsurvivors (table 2). However, these differences must be interpreted with caution, since a single significant variable may be strongly influenced by the others. Thus, we performed a multivariate analysis that has the ability to test the significance of each variable whilst controlling the other variables considered in the analysis. This type of analysis is compulsory for an appropriate interpretation of the data base.

Our results are very close to those of GILlESPIE $e t$ al. [18]. By studying 327 patients consecutively submitted to mechanical ventilation for more than $24 \mathrm{~h}$, these authors documented that the number of organs or systems on failure was the main factor predicting mortality. However, in this report, only univariate analysis was performed. The investigators emphasized the rare occurrence of death from untreatable hypoxaemia, since the death of only one out of 80 patients with acute lung injury was directly related to hypoxaemia. Similarly, Bell et al. [19] reported only two deaths attributable to refractory hypoxaemia in a series of 104 ARDS patients. In both studies, pulmonary infection was the main factor predisposing to acute respiratory failure. However, not all authors agree regarding the negative prediction power of hypoxia. For instance, PAPADAKIS and BROWNER [20], studying 78 noncardiac medical patients, found that the necessity for $\mathrm{FIO}_{2}>0.5$ at $24 \mathrm{~h}$ of mechanical ventilation was a predictor of mortality.

BARTLETT et al. [2] reported a series of ARDS patients treated with extracorporeal membrane oxygenation. These investigators could not find any physiological variable recorded on patients' admission useful to discriminate survivors from nonsurvivors. They concluded that the most important factor related to outcome in acute respiratory failure was the failure of other organs or systems. The prognosis worsened when the number of failing organs increased. MONTGOMERY et al. [4] studied 207 patients at risk for developing ARDS. There were only five deaths from irreversible respiratory failure. Most deaths occurring during the first $72 \mathrm{~h}$ after inclusion in the study were related to the underlying illness. In agreement with our results and coinciding with them, the APACHE score also showed a linear correlation with the probability of death. Likewise, similar results have been reported by FowLER et al. [3] in a well-designed study of 88 ARDS patients. They observed the absence of predictive value of a number of physiological variables (namely, $\mathrm{FIO}_{2}$, arterial carbon dioxide tension $\left(\mathrm{PaCO}_{2}\right)$, $\mathrm{PaO}_{2}$, oxyhaemoglobin saturation, tidal volume, maximal PEEP, dynamic and static compliance, and arterial and wedge pulmonary pressures) using powerful statistical tools, such as the multiple discriminant function analysis and the Cox proportional hazard method.

The influence of a possible bias because of the inclusion of short-term mechanically-ventilated patients in determining prognosis was also analysed in our series. The predictive values obtained with the discriminant function in the whole group and in those patients requiring mechanical ventilation 24 and $72 \mathrm{~h}$ after admission were very similar. Thus, the inclusion of the latter patients did not bias the accuracy of the initial prediction. Although gas exchange variables reached some importance as predictors in patients needing mechanical ventilation for more than 24 and $72 \mathrm{~h}$, both the NAC and SAPS remained as the best predictors. To the best of our knowledge, there is not yet any additional information regarding this issue in the literature.

Two additional characteristics of the patients' group in our study need to be borne in mind. Firstly, the population that we studied was not entirely uniform, since patients with different risk factors due to their underlying illness were included. It would be of great clinical interest to be able to identify groups of patients with high risk of dying by simple prediction rules [21,22]. Likewise, the inclusion of subjective impressions must be avoided because of their wide variability [23]. Secondly, 19 patients $(16 \%)$ had an acute exacerbation of chronic respiratory failure, another possible bias factor introduced in the analysis [24]. However, when a discriminant analysis excluding these patients was performed, the original results did not change significantly when compared to the whole population.

Although age is into SAPS, age alone was not a predictor of mortality. This unusual finding was repeatedly found in all the analyses performed. One possible explanation is that the population was too small and diverse. For instance, old patients with COPD or pneumonia had a good prognosis. An alternative explanation could be that in medical intensive care unit (ICU) patients, the influence of age on mortality is not so strong compared to surgical ICU populations. Other authors studying medical patients receiving mechanical ventilation have found similar results [20]. In a recent paper from our group devoted to studying the prognosis factors of 92 cases of severe community-acquired pneumonia, age was not one of the predictors of mortality selected by a multivariate analysis [25]. Indeed, further studies are warranted to confirm this important finding.

We conclude that the number of associated complications on admission and the simplified acute physiological score were the main determinants of outcome in a general population of patients submitted to mechanical ventilation. Hypoxaemia and related parameters were useless in predicting the outcome. Although respiratory therapy, especially mechanical ventilation, can provide a safe arterial oxygenation, therapeutic interventions on other organs and systems and the prevention of infection seems to play a key role for improving and optimizing the prognosis of patients with acute respiratory failure in the intensive care setting.

\section{References}

1. Pontoppidan $\mathrm{H}$, Geffin B, Lowenstein E. Acute respiratory failure in the adult. $N$ Engl J Med 1972; 287: 743-752. 
2. Bartlett RH, Morris AH, Fairley HB, Hirsch R, O'Connor $\mathrm{N}$, Pontoppidan $\mathrm{H}$. A prospective study of acute respiratory failure. Chest 1986; 89: 684-689.

3. Fowler AA, Hamman RF, Zerbe GO, Benson KN, Hyers TM. Adult respiratory distress syndrome: prognosis after onset. Am Rev Respir Dis 1985; 132: 485-489.

4. Montgomery AB, Stager MA, Carrico J, Hudson L. Causes of mortality in patients with the adult respiratory distress syndrome. Am Rev Respir Dis 1985; 132: 485489.

5. Traver GA, Cline M, Burrows B. Predictors of mortality in chronic obstructive pulmonary disease. A 15 year follow-up study. Am Rev Respir Dis 1979; 119: 895902.

6. Asmundsson T, Kilburn KH. Survival after acute respiratory failure: 145 patients observed 5 to 8.5 years. Ann Intern Med 1974; 80: 54-57.

7. Gottlieb LS, Balchum OJ. Course of chronic obstructive pulmonary disease following first onset of respiratory failure. Chest 1973; 63: 5-8.

8. Martin TR, Lewis SW, Albert RK. The prognosis of patients with chronic obstructive pulmonary disease after hospitalization for acute respiratory failure. Chest 1982; 82: 310-314.

9. Kaelin RM, Assimacopoulos A, Chevrolet JC. Failure to predict six-month survival of patients with COPD requiring mechanical ventilation by analysis of simple indices. Chest 1987; 92: 971-978.

10. Jardin F, Prost JF, Bazin M, Desfond P, Ozier Y, Margairaz A. Modalités évolutives du syndrome de détresse respiratorire aigüe de l'adulte: valeur pronostique d'un indice de gravité tiré de l'oxygenation arteriélle. Nouv Presse Méd 1982; 11: 29-33.

11. Johanson WG, Pierce AK, Sandford JP, Thomas GD. Nosocomial respiratory infections with Gram-negative bacilli. The significance of colonization of the respiratory tract. Ann Intern Med 1972; 77: 701-706.

12. Pepe PE, Reus DH, Hudson LD, Carrico CJ. Clinical predictors of the adult respiratory distress syndrome. Ann Intern Med 1983; 99: 293-298.
13. Le Gall JR, Brun-Buisson C, Trunet P, Latourniere J, Chatereau S, Rapin M. Influence of age, previous health status, and severity of acute illness on outcome from intensive care. Crit Care Med 1982; 10: 575-577.

14. Teasdale G, Jennet R. Assessment of impaired consciousness and coma. Lancet 1974; ii: 81-84.

15. Le Gall JR, Loirat P, Alperovitch A. Simplified acute physiologic score for intensive care patients. Lancet 1983; ii: 741 .

16. Cupples LA, Heeren T, Schatzkin A, Colton T. Multiple testing of hypothesis in comparing two groups. Ann Intern Med 1984; 100: 122-129.

17. Knaus WA. Prognosis with mechanical ventilation: the influence of disease, severity of disease, age, and chronic health status on survival from acute illness. Am Rev Respir Dis 1989; 140: S8-S13.

18. Gillespie DJ, Marsh HMM, Dvertie MB, Meadows C. Clinical outcome of respiratory failure in patients requiring prolonged mechanical ventilation. Chest 1986; 90: 364-369.

19. Bell RC, Coalson JJ, Smith JD, Johanson WG. Multiple organ system failure and infection in adult respiratory distress syndrome. Ann Intern Med 1983; 99: 293-298.

20. Papadakis MA, Browner WS. Prognosis of noncardiac medical patients receiving mechanical ventilation in a veterans hospital. Am J Med 1987; 83: 687-692.

21. Perkins HS, Jonsen AR, Epstein WH. Providers as predictors: using outcome predictions in intensive care. Crit Care Med 1986; 14: 105-110.

22. Perkins HS. Using outcome predictions to make treatment decisions. Chest 1987; 91: 475.

23. Pearlman RA. Variability of physician estimates of survival for acute respiratory failure in chronic obstructive pulmonary disease. Chest 1987; 91: 515-521.

24. Hudson L. Survival data in patients with acute and chronic lung disease requiring mechanical ventilation. Am Rev Respir Dis 1989; 140: S19-S24.

25. Torres A, Serra-Batlle J, Ferrer A, et al. Severe community-acquired pneumonia. Epidemiology and prognosis factors. Am Rev Respir Dis 1991; 144: 312-318. 